\title{
EEG to MRI Registration Based on Global and Local Similarities of MRI Intensity Distributions
}

\author{
Žiga Špiclin ${ }^{1}$, Arne Hans ${ }^{2}$, Frank H. Duffy ${ }^{2}$, Simon K. Warfield ${ }^{2}$, \\ Boštjan Likar ${ }^{1}$, and Franjo Pernuš ${ }^{1}$ \\ ${ }^{1}$ Faculty of Electrical Engineering, University of Ljubljana, Slovenia \\ \{ziga.spiclin, bostjan.likar,franjo.pernus\}@fe.uni-lj.si \\ 2 Department of Radiology, Children's Hospital Boston, USA \\ simon. warfield@childrens. harvard.edu
}

\begin{abstract}
In this paper, a novel method for EEG to MRI registration is proposed. Initial registration is achieved by extracting and matching symmetry planes of MRI and EEG data, followed by iterative registration based on minimizing a cost function. Comparison of the intensity distributions of the whole MR image and MRI voxels around a head surface point yields global similarities, while the comparison of intensity distributions of MRI voxels around corresponding EEG points, which reflects the head's sagittal symmetry, yields local similarities. Therefore, when the EEG points are registered to the MR image, maximal global and local similarities should be obtained. The cost function, incorporating global and local similarities, was the sum of Kullback-Leibler divergences between corresponding intensity distributions. The proposed method was evaluated on clinical MRI data with simulated EEG data, yielding mean registration error of $0.48 \pm 0.33 \mathrm{~mm}$, while with real EEG data an average root-mean-square point-to-surface error of $2.27 \pm 0.02 \mathrm{~mm}$ was obtained.
\end{abstract}

\section{Introduction}

Correlating functional information with anatomical localization offers the ability to understand how the brain functions. For in vivo neurophysiological studies of the brain, for example to localize the epileptogenic foci, magnetic resonance imaging (MRI) is usually integrated with functional information like electroencephalography (EEG), magnetoencephalography (MEG) or transcranial magnetic stimulation (TMS). EEG techniques, especially, allow for high-resolution measurements of temporal and, if related to brain anatomy derived from MRI, also spatial dimensions of brain's electrical activity 11. However, regarding the anatomical precision of clinically relevant locations of brain's electrical activity, accurate registration of MRI and EEG data is needed.

Retrospective registration of MRI and EEG, which is concerned with spatial localization of EEG electrodes in the MRI image, is usually solved by 1) extracting head surface from MR image, 2) acquiring EEG coordinates in physical space by some 3-D point localization technology and 3) using a surface-matching technique [2] to align the head surface and the localized 3-D coordinates of EEG 
electrodes. Previously published methods for EEG/MEG to MRI registration 34567] or for TMS to MRI registration [8] typically utilize the three abovementioned steps. However, using the head surface as the only feature of the information rich MRI data renders the registration process an ill-posed problem, especially due to the spherical symmetry of the head shape. Therefore, to get a well-behaved registration process, we propose to avoid segmentation of the head surface and choose to exploit the image intensities of the original MRI data.

In this paper, the main idea is to use MRI intensity information to drive the registration of MRI and EEG. Given a set of localized 3-D coordinates of EEG electrodes or EEG points that resemble the head's shape, we pursue to exploit the sagittal symmetry of the head by analyzing the topology of the EEG points, which yields the so-called local similarities. On the other hand, we also take account of the so-called global similarities, i.e. the similarities between the global intensity distribution and the intensity distribution of MRI voxels in the neighbourhood of each EEG point. By this approach a cost function is designed that yields a highly accurate and robust spatial registration of MRI and EEG.

\section{Materials and Methods}

In this section, a novel method for registration of MRI and EEG is presented. The outline of the proposed registration method is depicted in Fig. 1, Registration of the MRI and EEG input data is performed by: 1) computing the symmetry planes of MRI and EEG data and defining point-to-point correspondences of EEG points, 2) initial closed-form registration and 3) local iterative registration. In the following subsections, the MRI and EEG input data preprocessing and the initial and local EEG to MRI registrations are explained.

\subsection{MRI and EEG Datasets}

Three sets of MRI and EEG data were acquired for the present study. All patients were 8 to 10 years old volunteers, including normal controls and subjects born prematurely, enrolled in a separate ongoing study of the impact of premature birth on brain development. T1-weighted MR images were acquired by Siemens 3T Tim Trio MRI scanner using MPRAGE acquisition sequence with $18 \mathrm{~cm}$ FOV, $1.0 \mathrm{~mm}$ contiguous slice thickness, $256 \times 256$ sagittal slices covering the entire head, $\mathrm{TR} / \mathrm{TE}=1410 \mathrm{~ms} / 2.27 \mathrm{~ms}, \mathrm{TI}=800 \mathrm{~ms}$, flip angle $=9$ degrees, $\mathrm{iPAT}=2$. Scan time was approximately $3 \mathrm{~min} 40 \mathrm{~s}$. To acquire the EEG data, the EGI GES 250 system (www.egi.com) with HydroCel geodesic sensor net cap utilizing 128 EEG electrodes was used. The EEG sensor net cap was mounted on the patient's head and by using the EGI geodesic photogrammetry system, the $3-\mathrm{D}$ coordinates of EEG electrodes were digitized in a few seconds.

\subsection{MRI and EEG Data Preprocessing}

Prior to registration, the input MRI and EEG data is preprocessed to get an initial registration of MRI and EEG and to derive the corresponding pairs of 


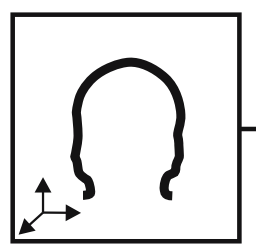

(a)

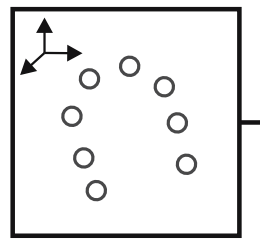

(c)

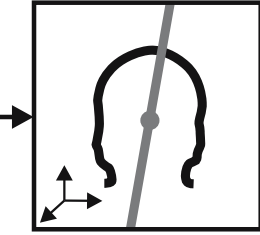

(b)

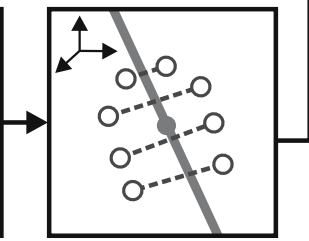

(d)

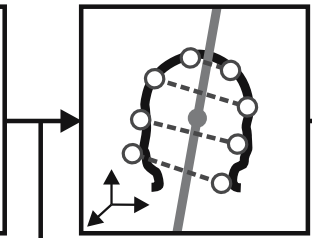

(e)

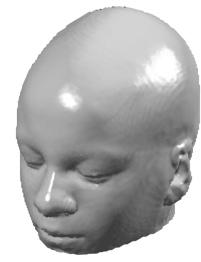

(g)

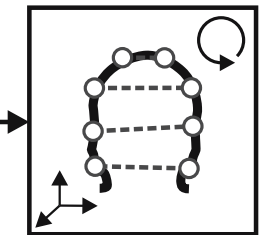

(f)

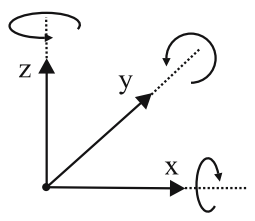

Fig. 1. Proposed method for registration of MRI and EEG: (a) input MR image and (b) the extracted mid-sagittal plane of the brain [9, (c) input EEG points and (d) the extracted symmetry plane and point-to-point correspondences of EEG points. An initial closed-form registration step (e) aligns the extracted symmetry planes of the MR image and the EEG points, followed by (f) local iterative registration procedure. The extracted head surface and corresponding MR image coordinate system $(\mathrm{g})$.

EEG points that reflect the sagittal symmetry of the head. To obtain an initial registration of MRI and EEG, we propose to extract and match the symmetry planes of both the MRI and the EEG points. The center of gravity and the symmetry plane of the MR image, also called the mid-saggital plane (MSP), are computed by the method of Ardekani et al. 9].

The symmetry plane of EEG points (ESP) is computed from its 3-D geometrical moments [6], i.e. the center of gravity and the principal axes derived from the inertia matrix of EEG points. Parameters of the ESP are estimated by using the center of gravity of EEG points as the plane origin and the cross-product between two principal axes, corresponding to the smallest and the largest eigenvalue of the inertia matrix, as the plane normal. Such an estimation of the ESP is valid without loss of generality, since the fixed and physically constrained configuration of the EEG sensor net cap preserves the distribution of EEG points for different patients. As the distribution of EEG points resembles the head shape of the patient, the ESP, therefore, corresponds to the MSP derived from MRI.

Let the ESP plane ${ }^{E S P} p(x, y, z)=0$ divide the EEG points $V=\left\{V_{i} ; i=\right.$ $1 \ldots N\}$ into subsets $\{V 1, V 2\}$ :

$$
V_{i} \in \begin{cases}V 1 & \text { if }{ }^{E S P} p\left(V_{i}\right) \geq 0 \\ V 2 & \text { otherwise }\end{cases}
$$

Using $\{V 1, V 2\}$ that contain $n_{1}$ and $n_{2}$ points $\left(n_{1}+n_{2}=N\right)$, respectively, a maximum cardinality bipartite graph [10] is constructed according to the sorting criterion: 


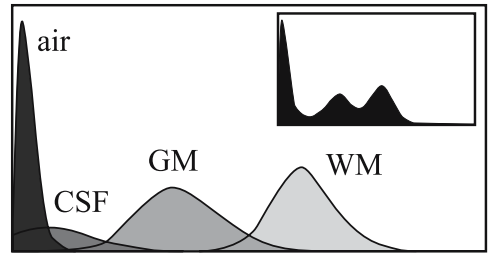

(a)

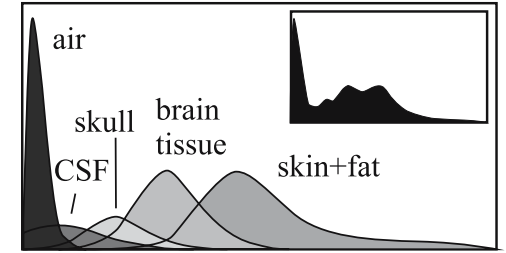

(b)

Fig. 2. Intensity distributions of (a) T1-weighted MR image and (b) local MRI voxels of a head surface point. Generally, modes of MR image intensity distribution correspond to air, cerebrospinal fluid $(C S F)$, grey matter $(G M)$ and white matter $(W M)$, while modes of local intensity distribution correspond to air, CSF, brain tissue, skin and fat.

$$
\left\{V 1_{i *}, V 2_{j *}\right\}=\underset{\substack{i=1 \ldots n_{1} \\ j=1 \ldots n_{2}}}{\operatorname{argmin}}\left\|V 1_{i}^{\perp}-V 2_{j}^{\perp}\right\|
$$

where points $V 1_{i}^{\perp}$ and $V 2_{j}^{\perp}$ correspond to $V 1_{i}$ and $V 2_{j}$, respectively, projected onto the ESP plane and $\|\cdot\|$ denotes the $L_{2}$-norm. In this way, the $N_{p} \leq N / 2$ corresponding point pairs $\left\{V 1_{i *}, V 2_{j *}\right\}$ reflect the sagittal symmetry of the head.

\subsection{Initial Closed-Form Registration}

Initial registration of MRI and EEG is obtained by first aligning the centers of gravity of the EEG points and the MR image, followed by a rotation that aligns the normals of MSP and ESP. The initial registration results in a coarse alignment of MRI and EEG, but incorporates a $\pi$ ambiguity around the $z$-axis and an undefined rotation around $x$-axis. The rotation ambiguities are resolved by minimizing, using a discrete angular step along the corresponding axes, the same cost function (4) that is used by the local iterative registration.

\subsection{Local Iterative Registration}

The initial registration is refined by the local iterative registration that uses a cost function based on the following two observations. First, the general shape of the T1-weighted MR image intensity distribution or global distribution (Fig. 2a) consists of four partially overlapping modes, corresponding to the air, cerebrospinal fluid (CSF), grey matter (GM) and white matter (WM). Second, a general local distribution, i.e. a distribution of intensities of MRI voxels around a head surface point (Fig. 2b), contains partially overlapping modes, corresponding to the air, CSF, skull, brain tissue, skin and fat. Computed from a large enough volume around a head surface point, the local intensity distribution is very similar to the global intensity distribution, because most of the image voxels correspond to the same anatomical structures (Fig. 2).

EEG points registered to the MRI should ideally lie on the head surface. Therefore, the local distributions corresponding to the registered EEG points 
should exhibit a high similarity to the global distribution. We call this similarity the global similarity. On the other hand, the local similarity is defined as the similarity between the local distributions of corresponding point pairs $\left\{V 1_{i *}, V 2_{j *}\right\}$ (Eq. 2). An optimal registration of MRI and EEG should yield maxima of both global and local similarities.

Instead of measuring the similarity, we measure the dissimilarity of two distributions $p_{1}$ and $p_{2}$ by the Kullback-Leibler divergence or relative entropy [11:

$$
K L\left(p_{1} \| p_{2}\right)=\sum_{i} p_{1 i} \log \frac{p_{1 i}}{p_{2 i}}
$$

Local intensity distributions are estimated by histograms, which are obtained by sampling a sphere of radius $r$ with a step $s$ along the $x-, y-$ and $z$-axes at locations of EEG points in the MRI image. Partial volume interpolation [12 is used to obtain the histograms of local intensity distributions, which results in a smooth cost function:

$$
C F=\sum_{k=1}^{N} K L\left(p\left(V_{k}\right) \| p_{g}\right)+\frac{N}{N_{p}} \sum_{k=1}^{N_{p}} K L\left(p\left(V 1_{i *(k)}\right) \| p\left(V 2_{j *(k)}\right)\right)
$$

with $p_{g}$ being the global intensity distribution and $p\left(V_{k}\right)$ being the local intensity distribution computed at point $V_{k}$. In (4), the first sum measures the global dissimilarity, while the second sum measures the local dissimilarity. Finally, optimal parameters for rigid registration of MRI and EEG are found by iteratively minimizing the cost function (4) using Powell's multi-dimensional directional set method and Brent's one-dimensional optimization algorithm [13].

\section{Experiments and Results}

Cost function validation. Histograms of local intensity distributions were obtained from a spherically shaped volume with radius $r=10 \mathrm{~mm}$ and sampling step $s=1 \mathrm{~mm}$. Histograms of both the local and global intensity distributions had 32 equally sized bins. Typical plots of the cost function (4) around the registered position of EEG points are shown in Fig. 3.

Registration results. Registration of MRI and EEG was evaluated on real data and by Monte Carlo simulations, as suggested by Singh et al. [14]. For this purpose, the head surface was extracted from MRI by interactive thresholding and manual correction (Fig. 1b). From the extracted head surface, 128 points were uniformly sampled to obtain the simulated EEG data. Transforming the simulated EEG points with a known rigid transformation and running the local iterative registration enabled the estimation of mean registration error (MRE), computed as a mean distance between the true and the registered positions of the simulated EEG points. By combined random translations and rotations of the simulated EEG, initial MREs were generated in the range of $[0,30] \mathrm{mm}$, with 10 misregistrations for each $1 \mathrm{~mm}$ MRE subinterval, yielding 300 starting 

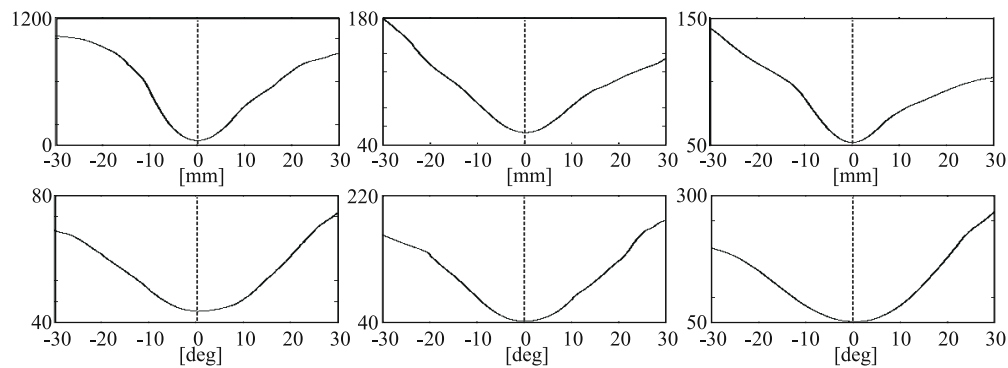

Fig. 3. Plot of the cost function (4) with respect to $x-, y-$ and $z-$ translations (top) and rotations (bottom) in the range of $[-30,30] \mathrm{mm}$ and $[-30,30]$ degrees, respectively, from registered position of EEG points
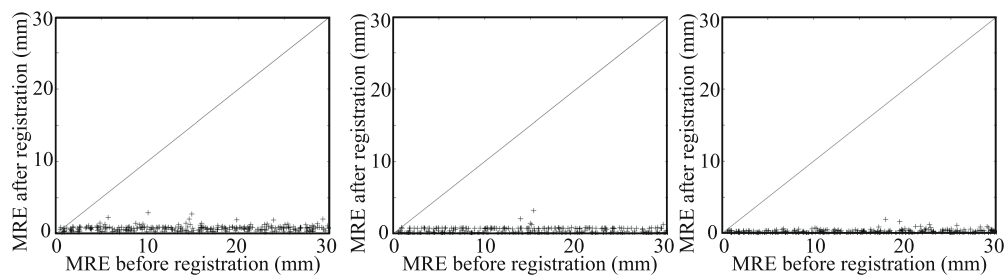

Fig. 4. Registration results with simulated EEG data for the three datasets, showing mean registration error ( $M R E$ ) of simulated EEG points before and after registration. By random translation and rotation of the simulated EEG points, 300 initial MREs were generated in the range of $[0,30] \mathrm{mm}$ MRE, with 10 misregistrations for each 1 mm subinterval.

Table 1. Registration results of the simulations and the patient's studies from the proposed and the previously published methods

\begin{tabular}{lll} 
Method & Simulation & Patient's study \\
\hline Schwartz el al. [3] & $0.37 \pm 0.15 \mathrm{~mm}$ & \\
Huppertz et al. [4] & & $3.39 \pm 0.24 \mathrm{~mm}$ \\
Brinkmann et al. [5] & $0.73-1.22 \mathrm{~mm}$ & $3.36 \pm 0.91 \mathrm{~mm}$ \\
Kozinska el al. 6] & $0.61 \pm 0.26 \mathrm{~mm}$ & $2.43 \pm 0.22 \mathrm{~mm}$ \\
Lamm et al. [7] & $0.17 \pm 0.30 \mathrm{~mm}$ & $1.17 \pm 0.38 \mathrm{~mm}$ \\
Noirhomme et al. 8] & $0.48 \pm 0.33 \mathrm{~mm}$ & $2.27 \pm 0.02 \mathrm{~mm}$ \\
Proposed method &
\end{tabular}

positions. After the local iterative registration, the obtained average MREs were $0.77 \pm 0.36,0.39 \pm 0.38$ and $0.28 \pm 0.24 \mathrm{~mm}$ for each of the three datasets. Fig. 4 shows Monte Carlo simulation results for the three datasets.

Using real EEG data, the initial closed-form registration was first executed. By a combined random translation and rotation in the range of $[-30,30] \mathrm{mm}$ and $[-30,30]$ degrees, respectively, 300 displacements of the initially registered 
EEG points were generated, followed by the local iterative registration. Rootmean-square error (RMS) was computed between the extracted head surface and the registered EEG data, yielding 2.41 $\pm 0.04,2.26 \pm 0.02$ and $2.14 \pm 0.01 \mathrm{~mm}$ for each of the three datasets. Computing the overall average RMS enabled us to compare our results to previously published results (Table 1).

\section{Discussion}

Accurate registration of MRI and EEG was obtained by employing MRI intensity information into the registration process. The proposed method initially aligned the MRI and EEG by extracting and matching the symmetry planes of MRI and EEG data. To refine the initial registration, the local iterative registration based on minimizing a cost function (4) was executed. The cost function was a sum of local and global similarities, expressed as the Kullback-Leibler divergences [11] between corresponding local and between local and global intensity distributions, respectively. Using only the global similarities as the cost function yielded successful registrations of MRI and EEG. However, employing local similarities improved the distinctivness of global minima for translation in $x$ - and rotation around $y-$ and $z$-axes, while unbiased global minima for the remaining parameters of rigid transformation were observed. Due to the smooth cost function (Fig. 3), the global minima was reached in 5-10 iterations, yielding an overall execution time $<2$ minutes, acceptable for a routine clinical use.

Results with simulated EEG data gave an average MRE of $0.48 \pm 0.33 \mathrm{~mm}$, while the results on the real EEG data gave an average RMS of $2.27 \pm 0.02 \mathrm{~mm}$. It is important to emphasize that with the proposed method, the evaluation metrics (MRE, RMS) are independent of the optimizing cost function (4), contrary to the methods 345678. Nevertheless, as Table 1 indicates, the results obtained by the proposed method are comparable to the results in 3445678 . The methods 34515 6 $7 / 8$ are exclusively based on the free-form surface matching techniques [2], that rely on proper segmentation of the head surface from MRI. According to Noirhomme et al. 8], registration errors due to segmentation of MRI can amount to as much as $1 \mathrm{~mm}$ in MRE and RMS. Due to the spherical symmetry of the head shape, more points than there are EEG electrodes are required by methods 3 345676 . This problem has been solved by using either spline interpolation of EEG points [7, acquiring more than 1000 virtual EEG points [3456] or applying a special point pattern during point acquisition [8, resulting in 200 800 virtual EEG points. Generally, the less points are used for the free-form surface matching, the poorer is the registration accuracy. Moreover, reducing the set of EEG points can lead to local minima in the distance measure, used in 345678 . The proposed method, on the other hand, uses only the original 128 EEG points. By exploiting the MRI intensities it yielded accurate registration of MRI and EEG. Besides, because the cost function contained no local minima (Fig. 3), the proposed registration method had a large capturing range $(>30$ $\mathrm{mm},>30$ degrees). 
Errors between the registered EEG points and corresponding true electrode coordinates in the MR image are not directly reflected by the RMS metric. In addition to registration inaccuracy, noise in the acquired EEG points and surface extraction errors contribute to the RMS. Thus, a ground truth for EEG to MRI registration, e.g. using MRI-visible markers attached to EEG electrodes, must be established to additionally validate the proposed, and any other, method.

To conclude, a fully automatic MRI intensity-driven method for registration of MRI and EEG was presented and evaluated on simulated and real EEG data. By incorporating MRI intensity information in the registration process, segmentation of MRI can be omitted without deteriorating the registration accuracy (Table 1). Moreover, using the framework of the proposed method (Fig. 1), other anatomical (CT) or functional (positron emission tomography and functional MRI) information can be correlated with modern point-based functional methods (EEG, MEG, TMS). In this way, the clinical neurophysiology would benefit from the ability of matching any tomographic data with the point-based functional methods [1, thus reducing cost and time of patient care.

Acknowledgements. This work has been supported by the Ministry of Higher Education, Science and Technology, Slovenia, under grants P2-0232, L2-7381, L29758, and J2-0716, in part by the NIH grants R01 RR021885, R01 GM074068 and R01 EB008015, and by a research grant from CIMIT.

\section{References}

1. Michel, C.M., Murray, M.M., Lantz, G., Gonzalez, S., Spinelli, L., de Peralta, R.G.: EEG source imaging. Clinical Neurophysiology 115(10), 2195-2222 (2004)

2. Audette, M.A., Ferrie, F.P., Peters, T.M.: An algorithmic overview of surface registration techniques for medical imaging. Medical Image Analysis 4(3), 201-217 (2000)

3. Schwartz, D., Lemoine, D., Poiseau, E., Barillot, C.: Registration of MEG/EEG data with 3D MRI: Methodology and precision issues. Brain Topography 9(2), 101-116 (1996)

4. Huppertz, H.J., Otte, M., Grimm, C., Kristeva-Feige, R., Mergner, T., Lucking, C.H.: Estimation of the accuracy of a surface matching technique for registration of EEG and MRI data. Electroencephalography and Clinical Neurophysiology 106(5), 409-415 (1998)

5. Brinkmann, B.H., O'Brien, T.J., Dresner, M.A., Lagerlund, T.D., Sharbrough, W., Robb, R.A.: Scalp-recorded EEG localization in MRI volume data. Brain Topography 10(4), 245-253 (1998)

6. Kozinska, D., Carducci, F., Nowinski, K.: Automatic alignment of EEG/MEG and MRI data sets. Clinical Neurophysiology 112(8), 1553-1561 (2001)

7. Lamm, C., Windischberger, C., Leodolter, U., Moser, E., Bauer, H.: Co-registration of EEG and MRI data using matching of spline interpolated and MRI-segmented reconstructions of the scalp surface. Brain Topography 14(2), 93-100 (2001)

8. Noirhomme, Q., Ferrant, M., Vandermeeren, Y., Olivier, E., Macq, B., Cuisenaire, O.: Registration and real-time visualization of transcranial magnetic stimulation with 3-D MR images. IEEE Transactions on Biomedical Engineering 51(11), 1994 2005 (2004) 
9. Ardekani, B.A., Kershaw, J., Braun, M., Kanno, I.: Automatic detection of the midsagittal plane in 3-D brain images. IEEE Transactions on Medical Imaging 16(6), 947-952 (1997)

10. Kreyszig, E.: Advanced Engineering Mathematics, 9th edn. John Wiley and Sons, Hoboken (2006)

11. Kullback, S., Leibler, R.A.: On information and sufficiency. The Annals of Mathematical Statistics 22(1), 79-86 (1951)

12. Maes, F., Collignon, A., Vandermeulen, D., Marchal, G., Suetens, P.: Multimodality image registration by maximization of mutual information. IEEE Transactions on Medical Imaging 16(2), 187-198 (1997)

13. Press, W.H., Teukolsky, S.A., Vetterling, W.T., Flannery, B.P.: Numerical recipes in C: The art of scientific computing. Cambridge University Press, Cambridge (1992)

14. Singh, K.D., Holliday, I.E., Furlong, P.L., Harding, G.F.A.: Evaluation of MRIMEG/EEG co-registration strategies using Monte Carlo simulation. Electroencephalography and Clinical Neurophysiology 102(2), 81-85 (1997) 